\title{
The Mode-Separated Pulse Profiles of Pulsar Radio Emission
}

\author{
Mark M. McKinnon \\ National Radio Astronomy Observatory, Green Bank, WV 24944 USA \\ Daniel R. Stinebring \\ Department of Physics, Oberlin College, Oberlin, OH 44074 \\ $U S A$
}

\begin{abstract}
Two modes of orthogonal polarization are generally observed in the radio emission from pulsars. Essentially all attempts to calculate the average profiles of the individual modes have assumed that the modes occur separately. We have presented evidence, however, that the modes occur simultaneously. Therefore, we believe that "mode-separated" pulse profiles which have been published to date reveal little about the polarization properties of the individual modes.

Calculating mode-separated pulse profiles for superposed orthogonal modes is complicated by the unknown polarization properties of the individual modes. However, the simplest model of pulsar radio emission which can explain the wide variety in the emission's polarization is one in which the individual modes are completely polarized. We use our simple model to compute the average profiles of the orthogonal modes in PSR B $0525+21$. The total intensity of the two mode profiles resembles the overall pulse profile, and each profile is highly linearly polarized.
\end{abstract}

\section{Introduction}

Polarization observations of the individual pulses from pulsars often show that the emission preferentially occurs at two polarization position angles separated by 90 degrees (e.g. Manchester, Taylor, \& Huguenin 1975; Backer \& Rankin 1980; Stinebring et al. 1984, hereafter SCRWB). The emission in each polarization mode also seems to prefer a particular sense of circular polarization, indicating that the modes are orthogonally and elliptically polarized (Cordes, Rankin, \& Backer 1978).

One would like to study the average polarization properties of the individual modes because it is possible that they could be the fundamental components of the radio emission. But before one can calculate "mode-separated" pulse profiles, one must first understand how the modes interact. The modes could be superposed, meaning both occur at the same time, or they could be disjoint, meaning that when one mode is present the other is always absent. Literature published on this topic over the past two decades has generally assumed that modes are disjoint (e.g. Cordes, Rankin, \& Backer 1978; Rankin 1988; Gil, Snakowski, \& Stinebring 1991; Gil et al. 1992), but in this paper, we point out that the polar- 
ization data are consistent with the superposition of highly-polarized orthogonal modes. In Section 2, we cite the evidence for highly-polarized superposed modes. In Section 3, we show how mode-separated pulse profiles can be calculated by assuming that the modes are superposed and completely polarized and then calculate the mode profiles for the pulsar PSR B0525+21.

\section{The Evidence for Highly-Polarized Superposed Modes}

When orthogonal modes occur with nearly equal frequency, the degree of linear polarization observed in the emission can indicate how the modes interact. The fractional linear polarization of the emission produced by superposed orthogonal modes will always be low. The polarization of the emission produced by disjoint modes can be high or low depending upon the degree of polarization of the individual modes (Cordes, Rankin, \& Backer 1978).

The average linear polarization we actually observe in a pulsar is low where the orthogonal modes occur, suggesting that the modes are superposed (SCRWB; McKinnon \& Stinebring 1998, hereafter MS). Histograms of fractional linear polarization and position angle constructed from data recorded in single pulse observations also indicate that modes are superposed; the majority of data samples have low fractional polarization when both modes occur (SCRWB; MS). Furthermore, a uniform component appears in position angle histograms when both modes are also present. This is what one would expect from superposed modes with comparable linear polarization. The linear polarization of the combined emission is low and therefore comparable with the instrumental noise. The position angle of instrumental noise is completely random and that is the uniform component one observes (MS).

Histograms of fractional linear polarization can be used to constrain the degree of polarization of the individual modes. Despite the presence of the orthogonal modes, some data samples in histograms of fractional polarization are highly, if not completely, polarized (McKinnon \& Stinebring 2000). This is the fractional linear polarization after the superposition of the modes; therefore, it is a lower limit on the intrinsic fractional polarization of the individual modes. With such a high degree of linear polarization, and after accounting for the circular polarization, one can reasonably postulate that the individual modes are completely polarized.

\section{Mode-Separated Pulse Profiles}

We can model the polarization properties of the individual modes by assuming that they are superposed and completely polarized. The modes can be represented by two antiparallel vectors in the $\mathrm{Q}-\mathrm{V}$ plane ${ }^{1}$ of Poincaré space, where the Stokes parameter $\mathrm{Q}$ represents the linear polarization and Stokes $\mathrm{V}$ represents the circular polarization. Since we assume that the modes are completely

\footnotetext{
${ }^{1}$ An arbitrary vector in $\mathrm{Q}-\mathrm{U}-\mathrm{V}$, or Poincaré, space can be rotated to the $\mathrm{Q}-\mathrm{V}$ plane without loss of generality in the model. The rotation concentrates the linear polarization in the Stokes parameter $Q$ and sets the value of Stokes $U$ to zero.
} 
polarized, the amplitudes of the vectors are equal to the flux densities of the modes, $\mu_{1}$ and $\mu_{2}$. The degree of linear and circular polarization for each mode is defined by the angle, $2 \psi$, between the $\mathrm{Q}$-axis and the vector representing the dominant mode. Since the modes are superposed, the sum of the two vectors gives the polarization vector of the combined emission. This polarization vector has an amplitude equal to the difference in mode flux densities and points in the direction of the dominant mode vector. Therefore, the Stokes parameters Q and $\mathrm{V}$ of the combined emission are proportional to the difference between the mode flux densities. The total intensity, or Stokes parameter I, of the combined emission is simply the sum of the mode flux densities. The mean values of I, Q and $\mathrm{V}$ are, in principle, measurable quantities. The combination of the model and measurements produces three equations and three unknowns. They can be used to calculate the mode flux densities at each pulse longitude and thus compute the mode-separated average profiles.

$$
\begin{gathered}
\mu_{\mathrm{I}}=\mu_{1}+\mu_{2} \\
\mu_{\mathrm{Q}}=\left(\mu_{1}-\mu_{2}\right) \cos (2 \psi), \\
\mu_{\mathrm{V}}= \pm\left(\mu_{1}-\mu_{2}\right) \sin (2 \psi) .
\end{gathered}
$$

Figure 1 shows the mode-separated profiles for PSR B0525+21 calculated from equations 1 through 3 using data recorded by SCRWB. The most striking features we find in these profiles are their high linear polarization and their similarities to one another as well as to the overall average profile (see SCRWB). Both modes are active at all pulse longitudes which may indicate that both modes originate at the same location within the magnetic flux tube above the polar cap. The modes would occur at different pulse longitudes and have very different shapes if they originated at different locations. The similarity in the shapes of the mode profiles may also indicate that the modes are produced by the same emission mechanism. If the modes are produced by separate emission mechanisms, both mechanisms must be active across the entire pulse. A detailed discussion of the material presented in this paper can be found in McKinnon \& Stinebring (2000).

\section{References}

Backer, D. C. \& Rankin, J. M. 1980, ApJS, 42, 143

Cordes, J. M., Rankin, J. M., \& Backer, D. C. 1978, ApJ, 223, 961

Gil, J. A., Snakowski, J. K., \& Stinebring, D. R. 1991, A\&A, 242, 119

Gil, J. A., Lyne, A. G., Rankin, J. M., Snakowski, J. K., \& Stinebring, D. R. 1992, A\&A, 255, 181

Manchester, R. N., Taylor, J. H., \& Huguenin, G. R., 1975, ApJ, 196, 83.

McKinnon, M. M. \& Stinebring, D. R. 1998, ApJ, 502, 883 (MS)

McKinnon, M. M. \& Stinebring, D. R. 2000, ApJ, 529, in press

Rankin, J. M. 1988, ApJ, 325, 314

Stinebring, D. R., Cordes, J. M., Rankin, J. M., Weisberg, J. M., \& Boriakoff, V. 1984, ApJS, 55, 247 (SCRWB) 

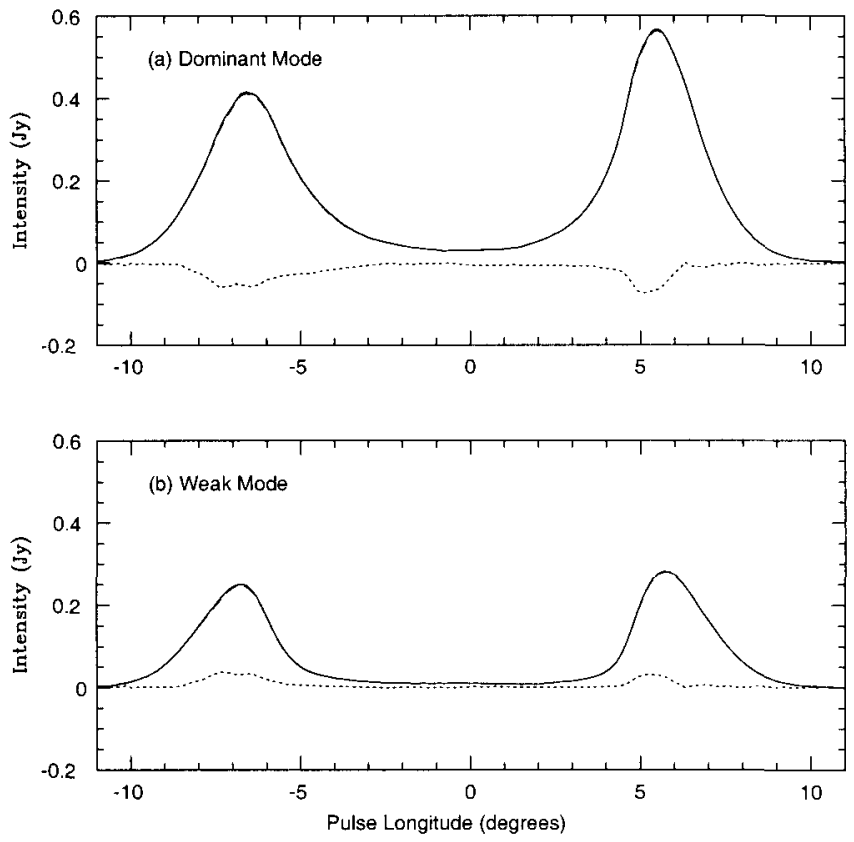

Figure 1. The average profiles of the superposed orthogonal modes in PSR B0525+21. The dominant mode profile is shown in (a), and the weak mode profile is shown in (b). The total intensity, linear polarization, and circular polarization of each profile are represented by the solid, dashed, and dotted lines, respectively. The dashed lines are almost completely obscured by the solid lines because each profile is almost completely linearly polarized. The two modes have opposite senses of circular polarization. 